\title{
Understanding the Obstacles and Influences in Adopting Infant Safe Sleep Practices
}

Alison Jacobson, Barb Himes, IBCLC

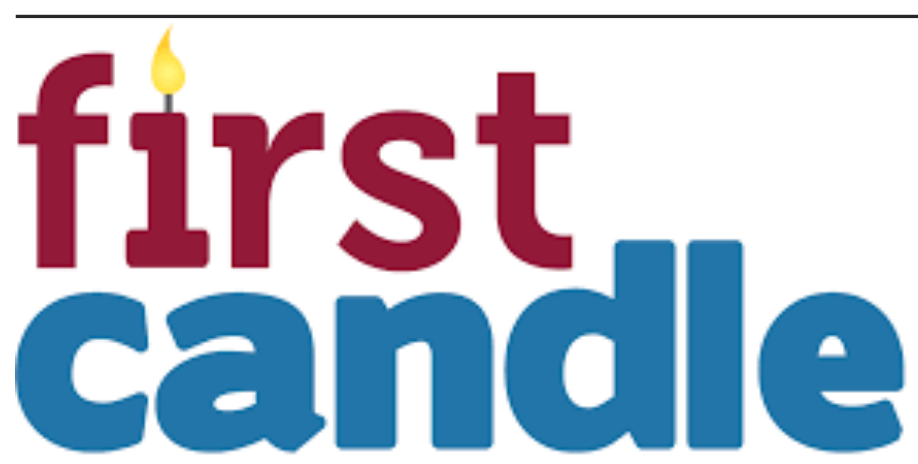

Saving babies. Supporting families.

First Candle's efforts to support families during their most difficult times and provide new answers to help other families avoid the tragedy of the loss of their baby are without parallel.

“Sudden Infants Death Syndrome (SIDS), making it the single leading cause of infant mortality in the U.S. Evidence indicates adherence to infant Safe Sleep Guidelines developed by the American Academy of Pediatrics (AAP) reduces these mortality rates."

\section{Abstract:}

Background: About 3,500 infants die annually within their first year of life from Sudden Unexplained Infant Death (SUID) and its subset Sudden Infants Death Syndrome (SIDS), making it the single leading cause of infant mortality in the U.S. Evidence indicates adherence to infant Safe Sleep Guidelines developed by the American Academy of Pediatrics (AAP) reduces these mortality rates. Still, since an initial decline following the 1994 guidelines introduction, SIDS rates have leveled, and SUID rates have increased. In addition, SUID rates per 100,000 live births are twice as high among Black and Native American infants than white. The AAP last updated the guidelines in 2016 and is scheduled to issue the next updated guidelines in late 2021.

Objective: To examine perceptions of the AAP guidelines held by parents and health care providers as they relate to guidelines comprehension and compliance, and to share this information with the AAP 2021 guidelines task force.

Methods: First Candle commissioned a qualitative research study in 2020, consisting of three online focus groups in three different states to identify perceptions and potential barriers to guidelines adoption by family members and providers, the outcomes of which were shared with the AAP for review as its guidelines task force develops the updated version to be released in 2021.

Results: Regarding how the AAP guidelines were viewed, there were responses across geographic and sociocultural variation regarding varying levels of general comprehension and trust in them, depending on the resource and acceptance of the language used. Additional factors were the emotional and practical realities of parenting, cultural and family traditions, and the belief that parental volition in decision-making is important.

Conclusion: Evidence-based protocols regarding infant safe sleep practices and their impact on reducing infant mortality rates may have proven value. Still, professional caregivers and family members feel that more cultural context and personal relevance are needed in presentation and communication if families are to trust and consider them for adoption and compliance.

\section{Keywords:}

infant mortality, infant safe sleep, implicit bias, Sudden Unexplained Infant Death (SUID).

\section{Definition of terms:}

Qualitative research: Information gathered first-hand regarding individual feelings, opinions, and perceptions, through focus group questions and discussion. The information is not quantified.

Purposive: qualitative research conducted with a sampling whose characteristics indicate it will likely have knowledgeable responses to the topics to be introduced.

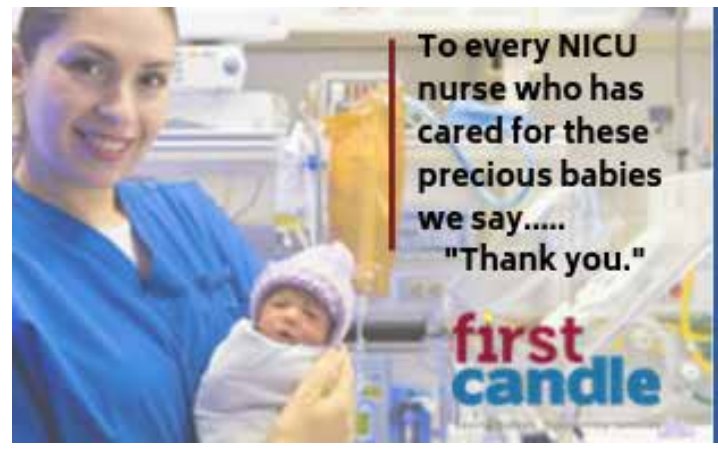

\section{Did you know that premature and low birth weight babies have a $4 \times$ greater risk for SIDS?}

At First Candle we're educating parents, grandparents and caregivers about safer sleep to make sure all babies reach their first birthday. Learn more at firstcandle.org 
Infant Safe Sleep Guidelines: the evidence-based recommendations developed by the American Academy of Pediatrics Task Force, to reduce the risk of Sudden Unexplained Infant Death, including Sudden Infant Death Syndrome and Accidental Suffocation and Strangulation in Bed (ASSB). The current revision in use was released in 2016.

Implicit bias: bias that can occur automatically, without conscious thought.

\section{"At the core of its Straight Talk for Infant Safe Sleep, train-the-trainer program is the AAP set of recommendations for infant safe sleep, last updated in 2016. (1) This version is the latest in a series dating to the original Back to Sleep campaign, which was developed by a coalition including NICHD, the AAP Task Force, NHLBI, HRSA, and the SIDS Alliance (now First Candle) in 1994."}

\section{Background:}

First Candle is a national 501c (3) whose mission is to eliminate Sudden Unexpected Infant Death Syndrome (SUID) and other sleep-related infant deaths while providing bereavement support for families who have suffered a loss. At the core of its Straight Talk for Infant Safe Sleep, train-the-trainer program is the AAP set of recommendations for infant safe sleep, last updated in 2016. (1) This version is the latest in a series dating to the original Back to Sleep campaign, which was developed by a coalition including NICHD, the AAP Task Force, NHLBI, HRSA, and the SIDS Alliance (now First Candle) in 1994. The recommendations focused on a supine infant sleep position on a firm flat surface, alone, with no extraneous bedding and no bed-sharing.

In 2011 the AAP expanded the guidelines to recommend regular prenatal care and breastfeeding for mothers and that infants should receive all recommended vaccinations.

Between 1994, when Back to Sleep (now Safe to Sleep®) began, and 1999, the overall SIDS rate in the U.S. dropped by more than $50 \%$. (2) However, since then, the national SIDS rate has changed little. One explanation is the classification has broadened into Sudden Unexpected Infant Death (SUID), which includes SIDS and accidental suffocation and strangulation in bed (ASSB), and deaths that might once have been considered SIDS are otherwise identified.

Although the causes of SIDS are still unknown and not preventable, the causes of ASSB are. Nonetheless, the CDC reports SUID is the single leading cause of death for infants one month to one year of age, currently resulting in an average of 3,500 infant deaths annually in the U.S. From 1990 to 2015, while SIDS rates decreased $35.8 \%$, ASSB rates increased $183.8 \%$ (3), and the rate is more than twice as high for Black, American Indian/Alaska Native babies as white babies.
Although compliance with the AAP guidelines can prevent infant death from ASSB, First Candle had become anecdotally aware, from its training work and collaboration with other maternal and infant health organizations, of resistance to adopting the recommendations, especially from communities of color. Reasons that were given included cultural preferences, socioeconomic realities, implicit bias within the language of the guidelines themselves, and bias in how health care professionals communicated the information.

Therefore, as the AAP task force prepared to develop the next set of updated guidelines, scheduled for release in 2021, First Candle commissioned a qualitative study to collect input from family members and health care providers regarding infant sleep practices and the AAP guidelines to help inform its work and to provide to the AAP task force, which agreed to review.

\section{Methods:}

First Candle commissioned research firms to conduct focus groups in Michigan, Georgia, and Connecticut, selected for geographical and statistical diversity. Per 100,000 live births, Michigan's SUID rate is 98.7; Georgia's is 124.7, and Connecticut's is 59.6.

The Michigan research was funded by a grant from the W.K. Kellogg Foundation. The Connecticut research was funded in part by a grant from The Community Foundation for Greater New Haven, and the Georgia research in part by a donation from Regal Lager, a Georgia-based distributor of infant and children's products. None of the funders had involvement in the study.

Each state study consisted of five separate sessions of 90 minutes to two hours, and participants included parents, grandparents, guardians, professional caregivers (nurses, doulas, midwives, and other healthcare workers), and breastfeeding mothers.

The first set of online focus groups was conducted in Michigan in October 2020, with a total sample size of 43. Age ranges were from 20 to $60+$ and geographies included Detroit Metro, North, Central, and Western Michigan, and the Upper Peninsula. Ethnicities included Black, Native American, Hispanic, White, and Asian.

The second set of online focus groups was conducted in Georgia in November 2020 with a sample size of 24. Age ranges were from 18 to 39, and geographies included Atlanta, Dublin, Macon, Pitts, and Cochran. Ethnicities included Black and White.

The third set of online focus groups was conducted in Connecticut in February 2021 with a sample size of 20. Age ranges were from 16 to 64, and geographies included Hartford, Norwalk, New Haven, Waterbury, and Stamford. Ethnicities included Black, Hispanic, Asian, and White.

The objective of this qualitative research was to:

- $\quad$ Determine the level of awareness about infant safe sleep practices among target audiences.

- $\quad$ Obtain feedback and reactions from the target audience to definitions of SUIDS/SIDS and related messaging statements.

- Identify and understand underlying factors that influence barriers to SIDS/SUIDS guidelines.

- Understand obstacles to safe sleep practices and guidelines among parents and care providers (doulas, agency, and private and public sector care provider program workers). 
- Understand obstacles and objections to safe sleep practices and guidelines as they relate to breastfeeding mothers.

- Gain insight into current practices, cultural beliefs, and behaviors related to safe sleep for babies.

"There was a purposive element in sample selection and question development. Through its training sessions and organizational outreach, First Candle has been anecdotally aware of the presence and effects of racial bias on maternal and infant health outcomes, including perceptions of the AAP safe sleep guidelines."

There was a purposive element in sample selection and question development. Through its training sessions and organizational outreach, First Candle has been anecdotally aware of the presence and effects of racial bias on maternal and infant health outcomes, including perceptions of the AAP safe sleep guidelines.

Therefore, it was considered essential to have racial diversity and discuss racial bias with families and care providers, including their own potential biases.

\section{Results:}

Overall, there was a general awareness about infant safe sleep, with understandably more knowledge about the guidelines from caregivers. There was greater awareness of SIDS than SUID and ASSB terminology, although parents recognize risks in suffocation. The language of the guidelines and the absence of cultural considerations were considered off-putting for some. Caregivers may choose to interpret the guidelines in what they feel are more understandable, contextual ways, such as La Leche League's Safe Sleep Seven, and may choose to defer to the mother's decisions regarding how and if they will be used.

Although some respondents may have agreed that the guideline statements are valid, they also may feel they are not always realistically achievable in daily life, including lack of access to resources to create this normative safe sleep environment and the challenges of work-life balancing.

"I think all moms... we've all been given advice. Baby needs to sleep in their own bed, whether it's a crib or a Pack and Play, firm mattress, tight-fitted sheet, no toys, no blankets, no pillows. A lot of times, you go into motherhood thinking, "I'm going to do it," but then when it comes down to it, it's like, this is not going to work." - Grandmother

"My baby sleeps right next to me, so all I have to do is literally roll over and pull out my breast. So, I'm not losing as much sleep. That's the reason why we chose that method, because if you have to get up and nurse, then that changes your level of exhaustion. You're more rested if you can just give the baby the breast in that way." - Breastfeeding Mother

"Books and doctors are almost the same thing. They're going to tell you what's in the books... They want you to listen to $a, b$, and c. But sometimes $a, b$, and c doesn't work for everybody.... I feel like if I can control it, I'm going to control it. - Mother

Fathers and mothers also may have different concerns about infant sleep practices, but the fathers will tend to defer to the mothers' wishes. Fathers may want to have a greater role in the decision-making on safe sleep but may feel they do not have sufficient information. Family and cultural influences will affect whether the guidelines are adopted and to what degree.

"I think my wife is obviously just naturally nurturing. I think I'm capable, but I always see she's a little more capable than I am. I think naturally women take on that role a little differently, so I think they're naturally better at it." - Father

"My boyfriend personally doesn't like [bedsharing], but l'm the one that gets up with the baby, so I feel like that's my decision if I want her to sleep in the bed with us or not." - Mother

Obstacles to adopting the AAP guidelines include family-held bedsharing beliefs, practices, and cultural influences that run counter to the guidelines, as well as uncertainty about messages from external resources and if they trust them. Therefore, alternatives such as the Safe Sleep Seven, which explores bed-sharing under certain conditions, may be considered.

Parenting is seen as deeply personal and cultural, shaped by experience and relationships with family, community, health care professionals, other care providers, and other parents. This may include feeling that making the infant sleep in a separate bed is a form of abandonment and that the infant is safer if the mother is nearby in bed.

\section{"Parenting is seen as deeply personal and cultural, shaped by experience and relationships with family, community, health care professionals, other care providers, and other parents. This may include feeling that making the infant sleep in a separate bed is a form of abandonment and that the infant is safer if the mother is nearby in bed."}

"America is like the highest rate of Sudden Infant Death Syndrome, but there are many, many other countries that sleep with their babies and babies don't die. So, I think that if it's done properly, it's a great thing for baby... it's huge for attachment developmentally for the baby." - Breastfeeding Mother

"As a breastfeeding mom, with your baby right there, you're constantly being affirmed throughout the night. Your baby is fine; your baby is alive and well to the point that they're able to latch and swallow and go back to sleep. That's comforting."- Breastfeeding Mother 
"I know it's dangerous because SIDS and stuff like that. But it's any time they move or cough, or it's like I'm right there and I feel like they're not right there with me it's like I couldn't get to him quick enough."- Breastfeeding Mother

Those following safe sleep practices may cite personal experience or how the information was presented, or an awareness that there may be newer information than what their parents knew.

"I definitely respect the advice from the pediatrician. I respectfully understand that I guess. But at the end of the day, I'm going to do what works best for me and my baby." - Mother

I'm not saying I don't trust my child's pediatrician, but white coat syndrome is real. She's a white physician; I'm going to just be honest. I'd rather go to my black medical professionals that I know." - Grandmother

"I ignored the doctor because I just thought it was something that couldn't happen to me. We're sleeping together, but we're safe. Then there was just that one night that I thought my husband rolled over on her, and I freaked out, and I said nope, she's got to go to her own crib. I just thought that wouldn't happen to me." - Mother

"I go to my mom, but also my mom is old school more because her kids are older, so a lot of her information is also outdated, so if she tells me something, sometimes l'll just Google it just to make sure that there's- it's not an outdated, don't do it anymore, like laying them on their bellies." - Mother

Parent-infant bonding and breastfeeding are considered essential, and guidelines that appear to interfere with that may be set aside, even if their safety intent is understood.

"I don't want to see my baby cry, so of course, I allow him to sleep in the bed with me. But does he need to be in a crib? Does he need to be his own safe space?"- Mother

"I'm scared of SIDS for real, and that's why I don't necessarily want her to sleep in her crib or in her bassinet. Because if she sleeps on my wife's arm or occasionally on my arm, I have control of how she's turned and how she can move." - Father

\section{"Understanding and consideration of them may depend on the interpersonal presentation and discussion with whoever is advocating them: medical professional, coach, social worker, doula, or other resources. The higher the level of trust in the messenger, the higher the chances are for guideline adoption. Trust is influenced by perceptions of racial or socioeconomic bias and a lack of meaningful two-way communication."}

\section{Conclusion:}

A" bottom line" assessment is that the context in which the AAP guidelines are conveyed is critical. The guidelines are less likely to be taken up by families as a standalone document. Understanding and consideration of them may depend on the interpersonal presentation and discussion with whoever is advocating them: medical professional, coach, social worker, doula, or other resources. The higher the level of trust in the messenger, the higher the chances are for guideline adoption. Trust is influenced by perceptions of racial or socioeconomic bias and a lack of meaningful two-way communication.

In addition, the greater the relevance the guidelines have to parents' lives, the greater the potential for them to be considered. The context reflects the realities of their lived experiences and examples that aid understanding. Clear and constructive communication that considers literacy levels and language needs could positively affect receptivity and potential adoption of the guidelines.

\section{Next Steps:}

The focus group study was commissioned as part of a larger initiative involving community task forces that can more effectively communicate infant safe sleep messages in more relevant ways and in line with families' lived experiences.

First Candle intends to work collaboratively with the AAP and the community task forces to deliver the new guidelines as they are released.

The desire would be for the task forces to represent both diverse and coalescent perspectives and to review and advise on how the revised guidelines could best be disseminated within their communities, both in terms of language, format, and interpersonal communications channels.

A digital media campaign would support this outreach with messages targeted to audiences within the infant ecosphere. It is expected that this could serve as a pilot for localized communications in regions across the country.

\section{References:}

1. SIDS and Other Sleep-Related Infant Deaths Updated 2016 Recommendations for a Safe Infant Sleeping Environment.

2. https://safetos/eep.nichd.nih.gov/activities/SIDS/progress

3. National and State Trends in Sudden Unexpected Infant Death: 1990 - 2015. Pediatric Volume 141, number 3, March 2018. https://pediatrics.aappublications.org/content/pediatrics/141/3/e20173519.full.pdf

Disclosure: The authors are affiliated with First Candle, Inc., a Connecticut-based not for profit 501(c)3 corporation.

NT 

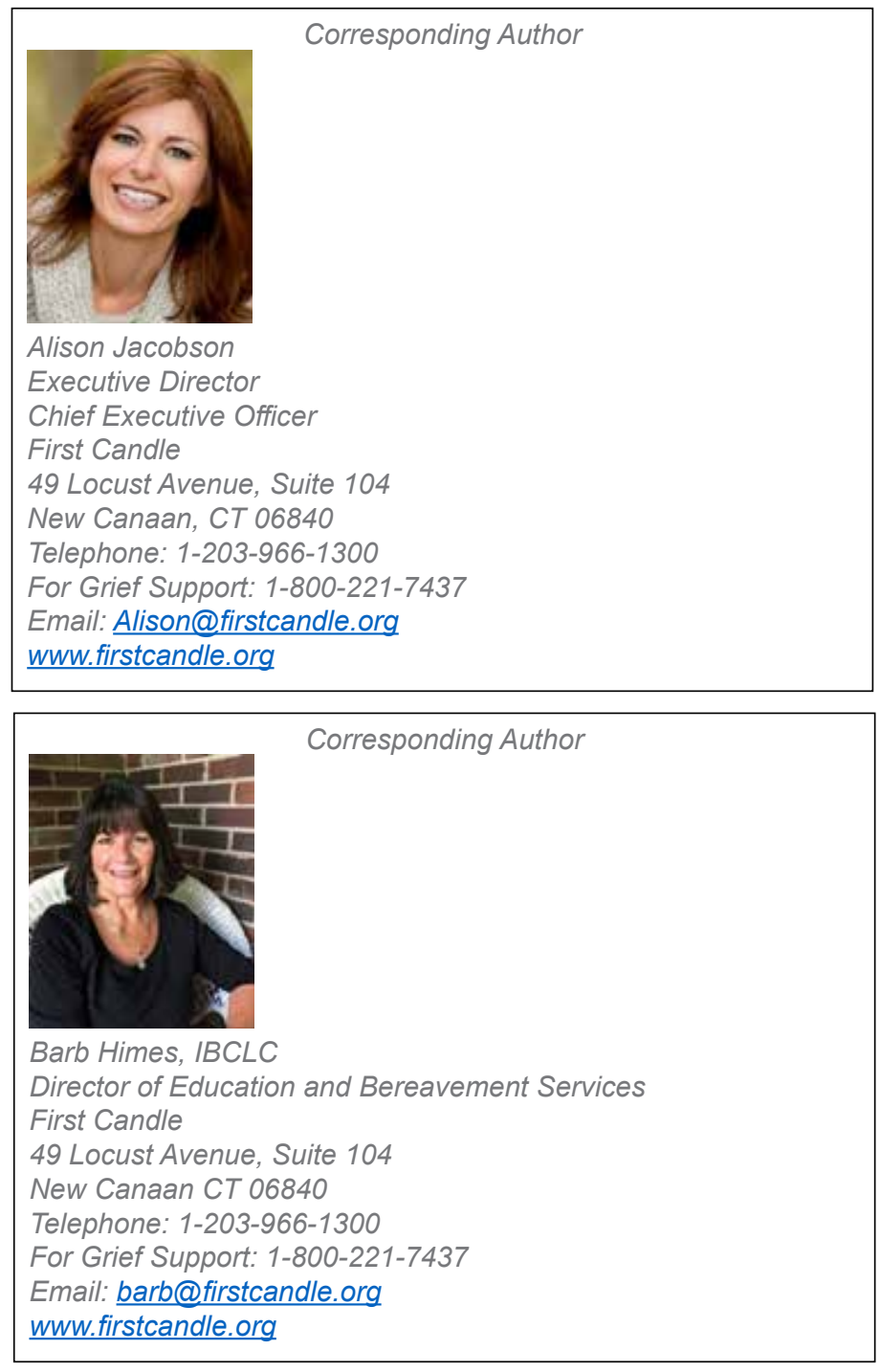

\section{About First Candle}

First Candle, based in New Canaan, CT, is a 501c (3) committed to eliminating Sudden Infant Death Syndrome and other sleeprelated infant deaths while providing bereavement support for families who have suffered a loss. Sudden unexpected infant death (SUID), which includes SIDS and accidental suffocation and strangulation in bed (ASSB), remains the leading cause of death for babies one month to one year of age, resulting in 3,600 infant deaths nationwide per year.
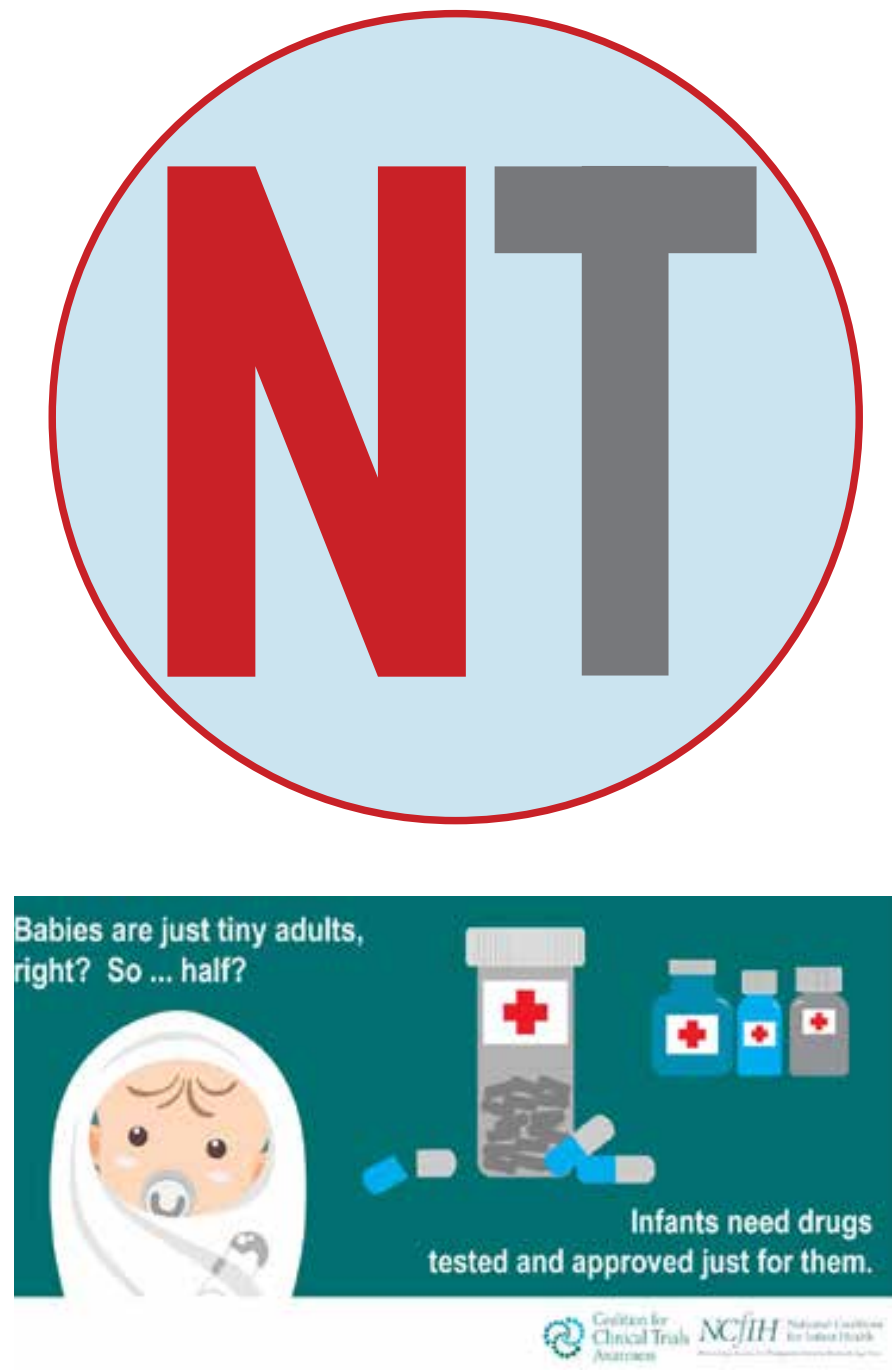

New subscribers are always welcome! NEONATOLOGY IQDAY

To sign up for a free monthly subscription, just click on this box to go directly to our subscription page

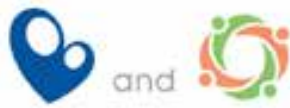

National Perinatal Association and NICU Parent Netwark

mynicunetwork.org 